\title{
First neutrino oscillation measurement in KM3NeT/ORCA
}

\section{Lodewijk Nauta, ${ }^{a, *}$ Valentin Pestel, ${ }^{a}$ Jerzy Mańczak, ${ }^{b}$ Dimitris Stavropoulos, ${ }^{c}$ Victor Carretero, ${ }^{b}$ Nhan Chau, ${ }^{d}$ Zineb Aly $^{e}$ and Paul de Jong ${ }^{a, f}$ on behalf of the KM3NeT Collaboration}

(a complete list of authors can be found at the end of the proceedings)

${ }^{a}$ Nikhef, Science Park 105, 1098XG Amsterdam, The Netherlands

${ }^{b}$ IFIC - Instituto de Fsica Corpuscular (CSIC - Universitat de València), c/Catedrático José Beltrán, 2 , 46980 Paterna, Valencia, Spain

${ }^{c}$ NCSR Demokritos, Institute of Nuclear and Particle Physics, Ag. Paraskevi Attikis, Athens, 15310 Greece

${ }^{d}$ Université de Paris, CNRS, Astroparticule et Cosmologie, F-75013 Paris, France

${ }^{e}$ Aix Marseille Univ, CNRS/IN2P3, CPPM, Marseille, France

${ }^{f}$ Universiteit van Amsterdam, Institute of Physics, Science Park 904, 1098XH Amsterdam, The Netherlands E-mail: 1jnauta@nikhef.nl

The KM3NeT/ORCA is a next-generation neutrino detector currently under construction in the Mediterranean Sea. There are currently 6 Detection Units deployed, and in the past year the detector has been steadily taking data. Here the first neutrino oscillation measurement is presented using data taken with the ORCA detector 6 Detection Units, containing 354.6 days of exposure. Selection criteria are discussed, followed by a neutrino oscillation analysis. In the analysis it is found that oscillations are preferred with a confidence level of $5.9 \sigma$ over "no oscillations". Likelihood scans of the $\Delta m_{31}^{2}$ and $\sin ^{2} \theta_{23}$ parameter also show a strong exclusion of the no oscillation hypothesis. The sensitivity contour in $\left(\sin ^{2} \theta_{23}, \Delta m_{31}^{2}\right)$ is presented, showing results that are approaching to being being competitive with other experiments [1].

$37^{\text {th }}$ International Cosmic Ray Conference (ICRC 2021)

July 12th - 23rd, 2021

Online - Berlin, Germany

\footnotetext{
*Presenter
} 


\section{Introduction}

The KM3NeT research infrastructure is currently building 2 next-generation neutrino detector at the bottom of the Mediterranean Sea at $\gtrsim 2.5 \mathrm{~km}$ depth. The ARCA detector is used for astrophysical studies of neutrino sources while the ORCA detector is used for studying neutrino oscillations with the ultimate goal of measuring the Neutrino Mass Ordering (NMO). The latest sensitivity studies to the NMO for the full detector are available at [2]. The ORCA detector is being built off the coast of France near Toulon, and will have an instrumented volume of 7Mton [2], while the ARCA detector will encompass approximately one cubic kilometre of sea water [3].

The ORCA detector will consist of 115 Detection Units (DU) containing 18 Digital Optical Modules (DOM) each. A DU is a vertical structure that houses the DOMs using cabling and supplies power and fibre-optics for read-out of the data taken by the DOMs. A DOM is a pressure resistant glass-sphere containing 31 3" photo-multiplier tubes (PMT), effectively creating a 3-dimensional grid of light sensors in the deep-sea. The detection principle is based on the Cherenkov effect: charged particles moving faster than the local speed of light emit Cherenkov radiation, which is detected by the PMTs. This allows for reconstruction of the interaction vertex, energy, direction and event topology, in turn allowing the probing of the phase-space of neutrinos interacting in the water where they create charged particles through weak interactions. The topology can either be track-like for outgoing muons at energies larger than a few $\mathrm{GeV}$ or shower-like for all other neutrino channels.

The ORCA detector is designed to probe an energy range of $1-100 \mathrm{GeV}$, at which atmospheric neutrinos dominate the neutrino flux. The reconstructed incoming angle $\theta_{\text {reco }}$ of the particle provides the baseline $L$ of the neutrino, and combined with the reconstructed energy $E_{\text {reco }}$ the oscillation phase space can be probed with the $L / E$ parameter. In this energy range it then becomes possible to measure the oscillation parameters $\theta_{23}$ and $\Delta m_{31}^{2}$ through the interaction rates of the neutrinos in the sea water.

\section{Data taking and selection criteria}

\subsection{Data taking}

The ORCA detector, has been steadily measuring in the deep sea since mid-2019, while it's being constructed. In 2019 ORCA consisted of 4 lines to form ORCA4, and in January of 2020 the detector was expanded by 2 lines to form ORCA6. The total data taking time for ORCA6 is 385.8 days.

\subsection{Selection criteria}

There are multiple selection criteria: for selecting runs to use in the analysis, for containing the events near the detector, selecting on reconstruction quality of the event and physics cuts to probe the relevant part of the phase-space. All with the goal of selecting a set of neutrino candidates to be used for analysis.

The run selection is based on the duration of a physics run, timing accuracy of the run, and the trigger rate. The runs that pass the selection criteria are considered to be of sufficient quality for physics analysis. For the ORCA6 data set this results in an exposure of 354.6 days. 
From these runs, neutrino candidates are selected to provide a sample to be used for the physics analysis. The overwhelming majority of recorded events are atmospheric muons, which have to be rejected in this process. To remove these events, cuts based on reconstruction quality, containment of the event in a volume around the detector and simple cuts to reject noise and hardware issues are applied to all reconstructed events. The cuts used in this analysis are set in such a way to reject as many atmospheric muons as possible, while allowing a subset of neutrinos to pass the cuts. In this way a relatively clean neutrino signal is recovered, while balancing to maintain good enough statistics to perform the physics analysis. Note the cut on reconstructed direction $\cos \theta_{\text {zenith }}$ in figure 1, which comes from oscillation physics, and is to ensure the neutrinos in the sample covered enough distance to allow for oscillations to a different flavour from the initial state.

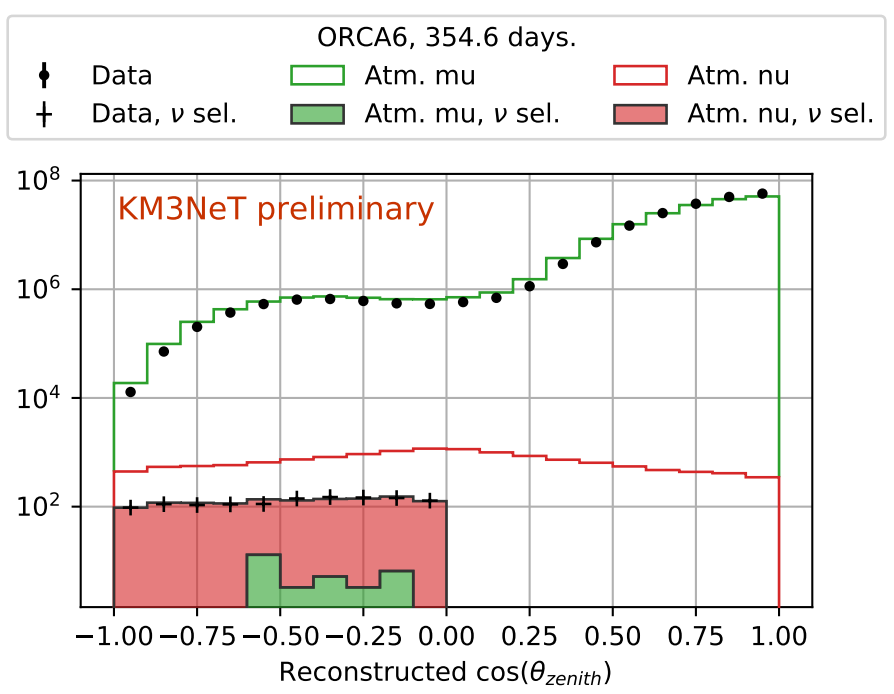

Figure 1: Incoming angle distribution of the selected data and MC sets used to do the analysis. The transparent distributions are before applying the cuts and the opaque distributions are after applying the cuts.

In figure 1, the event distributions before and after applying the selection criteria can be seen. The data and MC events in the figure are all after passing the initial run selection. In transparent green the atmospheric muon background is shown, which is several orders of magnitude larger than the neutrino flux in transparent red. In black the ORCA6 data is shown, showing in a good agreement between data and MC. The MC distributions are created from events generated with gSeaGen [4] and MUPAGE [5], which are subsequently propagated, triggered and reconstructed with KM3NeT software Jpp [3]. The distributions after applying all selection criteria are visible in the opaque distributions. In these distributions a small amount of atmospheric muons pass the cuts, while a significant fraction of the neutrinos pass too. This gives an S/B value of about 40 . These are the events that are used in the analysis.

In figure 2, the plots of the event distributions of the observables in the detector are shown, after applying the selection criteria. For the $\cos \theta$ figure this is the same distribution as the opaque distribution shown in figure 1.

Both distributions show good agreement between the measured data and the modelled MC, where oscillations use the parameter values according to NuFIT [1] normal ordering (NO), to 

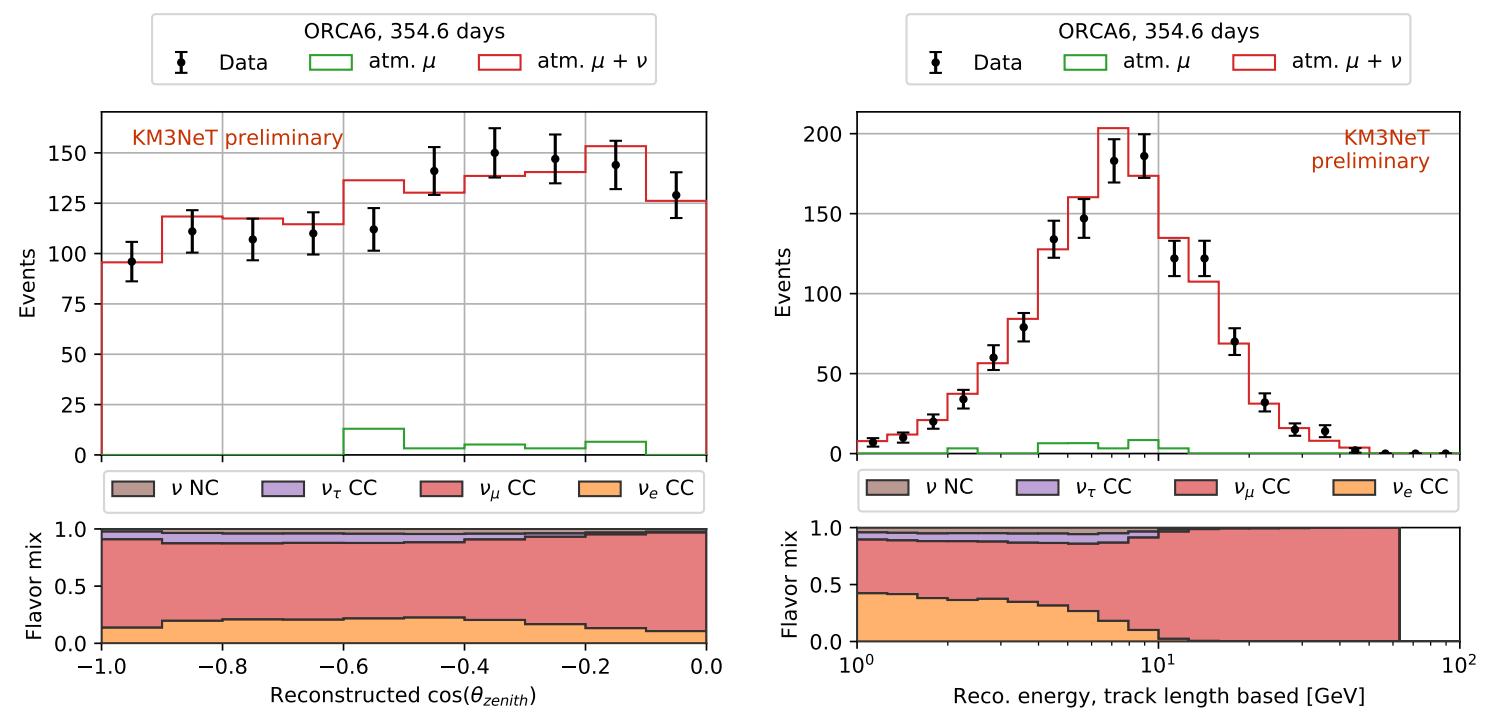

Figure 2: Left: Reconstructed incoming angle distribution after applying the cuts for both data and MC. Right: Reconstructed energy distribution after applying the cuts for both data and MC. Both figures show ORCA6 data and MC.

account for the oscillations that are present. Inverted ordering (IO) parameters have not been considered due to the detector not being sensitive to the NMO at its current size. The make up of the distribution per flavour is shown by stacking each channel in the lower part of the figure, and it can be seen that the $v_{\tau}$ particles start to appear at angles from $\cos \theta_{\text {reco }} \approx-0.2$ downward, which is consistent with our current understanding of oscillations. The measured events have only been reconstructed under a track-like hypothesis, while most of the events are known to result in shower-like topology. For this reason the sample is dominated by $v_{\mu}$-CC events, as the selection criteria use the reconstruction quality of the track hypothesis. The peak at $10 \mathrm{GeV}$ in the right part of figure 2 is due to the size of the detector. With only 6 lines in operation, only a fraction of the total light emitted is captured, limiting the accuracy of the reconstructed energy. The tail going up to about $50 \mathrm{GeV}$ is due to events moving in a more vertical direction up to the completely upward-going direction, where most light can be captured.

\section{Measuring neutrino oscillations}

The selected data set from ORCA6 has been used to do a study to find the neutrino oscillation parameters. In this section the oscillation parameters $\Delta m_{31}^{2}$ and $\theta_{23}$ are constrained and a contour plot of the $90 \%$ confidence level (CL) is shown, including systematic uncertainties.

\subsection{Measurement of oscillations}

For determining the oscillation parameters a negative log-likelihood minimization method is applied on events measured in the observable phase-space of reconstructed energy-incoming angle: $\left(E_{\text {reco }}, \cos \theta_{\text {reco }}\right)$. The incoming angle directly translates to the distance travelled of the initial 


\begin{tabular}{ll}
\hline & Treatment \\
Parameter & Free/Fixed/Prior \\
\hline$\theta_{12}[\mathrm{deg}]$ & Fixed \\
$\theta_{13}[\mathrm{deg}]$ & Fixed \\
$\theta_{23}[\mathrm{deg}]$ & Free \\
$\Delta m_{31}^{2}\left[10^{-3} \mathrm{GeV}^{2}\right]$ & Free \\
$\Delta m_{21}^{2}\left[10^{-5} \mathrm{GeV}^{2}\right]$ & Fixed \\
$\delta_{C P}[\mathrm{deg}]$ & Fixed \\
\hline Normalisation & Free \\
Spectral index & Prior: $10 \%$ \\
$n_{v_{\text {up }}} / n_{v_{\text {horiz }}}$ & Prior: $7 \%$ \\
$n_{v_{\mu}} / n_{v_{\bar{\mu}}}$ & Prior: $10 \%$ \\
$n_{v_{e}} / n_{v_{\bar{e}}}$ & Prior: $10 \%$ \\
$n_{v_{\mu}} / n_{v_{e}}$ & Prior: $3 \%$ \\
$n^{N C}$ & Prior: $10 \%$ \\
$n_{\tau}^{C C}$ & Prior: $20 \%$ \\
Energy scale & Prior: $10 \%$ \\
\hline
\end{tabular}

Table 1: The treatment of all oscillation and nuisance parameters in used the analysis. All priors are Gaussian and the values are relative to their nominal values.

neutrino through $L=R_{\text {Earth }} \cos \theta_{\text {reco, }}$, giving a handle on the oscillation probability. The log of likelihood

$$
\mathcal{L}=\prod_{i \in\left\{E_{\mathrm{reco},}, \cos \theta_{\mathrm{reco}}\right\}} P(d \mid m(\vec{p}, \vec{r}))
$$

is minimized for oscillation parameters $\vec{p}$ and nuisance parameters $\vec{r}$ provided to model $m$ which predicts the expected number of observed events in region $i$ of $\left(E_{\text {reco }}, \cos \theta_{\text {reco }}\right)$, given measured number of events $d$ in this region using Poisson probability $P$. The nuisance parameters $\vec{r}$ are described in detail in [8].

The likelihood is evaluated only for the ORCA6 track channel, as mentioned. An overview of all oscillation and nuisance parameters and their treatment can be found in table 1, where the nominal values of the oscillation parameters are determined by Nu-Fit 5.0 values [1].

A normalisation parameter is applied to the entire event distribution during the minimisation. This is done to capture unforeseen systematic uncertainties on the largest scale. The priors used are all applied as Gaussians and the input comes from either [2], [6] or [7], or are relaxed versions of values extracted from these references. The relaxation is done to account for the lower reconstruction accuracy and oscillation sensitivity of the partial detector compared to the full detector as described in [2]. 


\subsection{Results}

As a first step to find neutrino oscillations, the model $m$ is fitted to the data set in terms of reconstructed energy and incoming angle. The outcome of the fit is then transformed to $L / E$ and normalised compared to the "no oscillations" hypothesis for visualisation purposes. The model is also fitted against the data while constraining the oscillation parameters to either Nu-Fit 5.0 [1] values or "no oscillations" values while only marginalising the nuisance parameters.

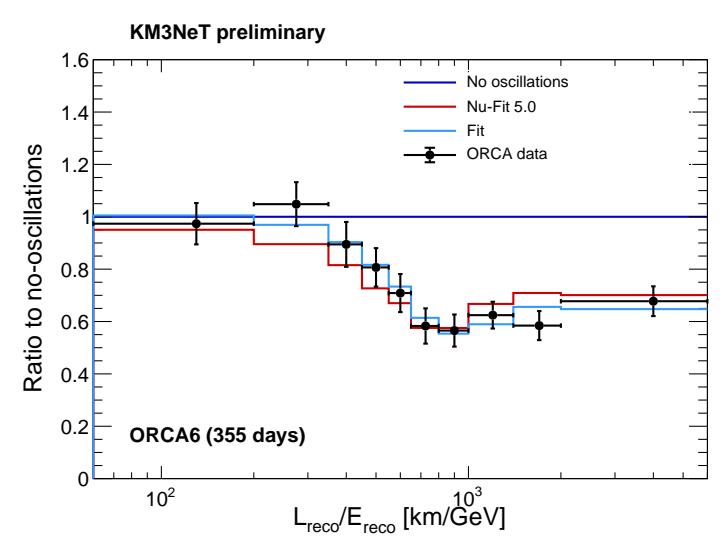

Figure 3: L/E distribution for the ORCA6 data and expected number of events relative to the "no oscillation" hypothesis. The binning is chosen such that similar statistics is present in each bin. The no oscillations and nu-fit curves in this figure do not include systematic uncertainties as modelled for the 'Fit' curve.

Figure 3 shows the $L / E$ distribution of the ORCA6 data, where the excellent agreement can be seen for oscillations. From the fit results the $\Delta \chi^{2}$ of the model is calculated and the significance of no oscillations compared to oscillations is estimated at $5.9 \sigma$, while having a difference with Nu-Fit of about $1.9 \sigma$.

The phase-space of oscillation parameters has also been scanned and profiled in terms of the negative log-likelihood, to provide 1-dimensional scans and a 2-dimensional contour of the sensitivity of the ORCA detector towards neutrino oscillations.

Figure 4 shows the 1-D scans of the profile likelihood with best fit values for the oscillation parameters added at the bottom. This is done for parameters $\sin ^{2} \theta_{23}$ and $\Delta m_{31}^{2}$. The best fit values for the parameters are $\sin ^{2} \theta_{23}=0.50_{-0.10}^{+0.10}$ (stat.+syst.) and $\Delta m_{31}^{2}=1.95_{-0.22}^{+0.24}$ (stat.+syst.), where the statistical and systematic error is given in its entirety. This best fit value is also shown in the contour in figure 5 .

Finally the contour with the sensitivity to the oscillation parameters $\theta_{23}$ and $\Delta m_{31}^{2}$ is shown in figure 5, in terms of $\sin ^{2} \theta_{23}$ for the former. At every point in the contour the log-likelihood is minimized relative to all nuisance parameters including the priors from table 1 , and the $90 \% \mathrm{CL}$ contour is drawn through the recovered likelihood landscape.

Figure 5 shows the contour of the analysis of ORCA6. The Nu-Fit best fit point is within the confidence level of $90 \%$ for this result. With almost year of data for ORCA6 the result is in the same order of magnitude as competing experiments, showing promise for future measurements with the ORCA detector. 

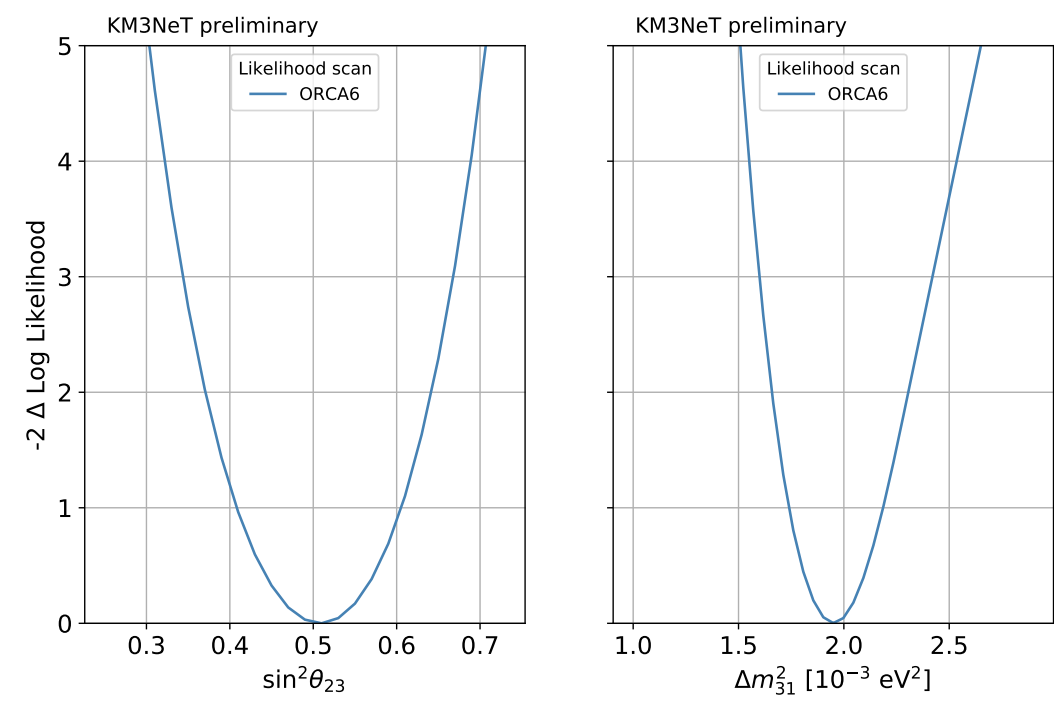

Figure 4: The profiled likelihood scan of $\sin ^{2} \theta_{23}$ (left) and $\Delta m_{31}^{2}$ (right).

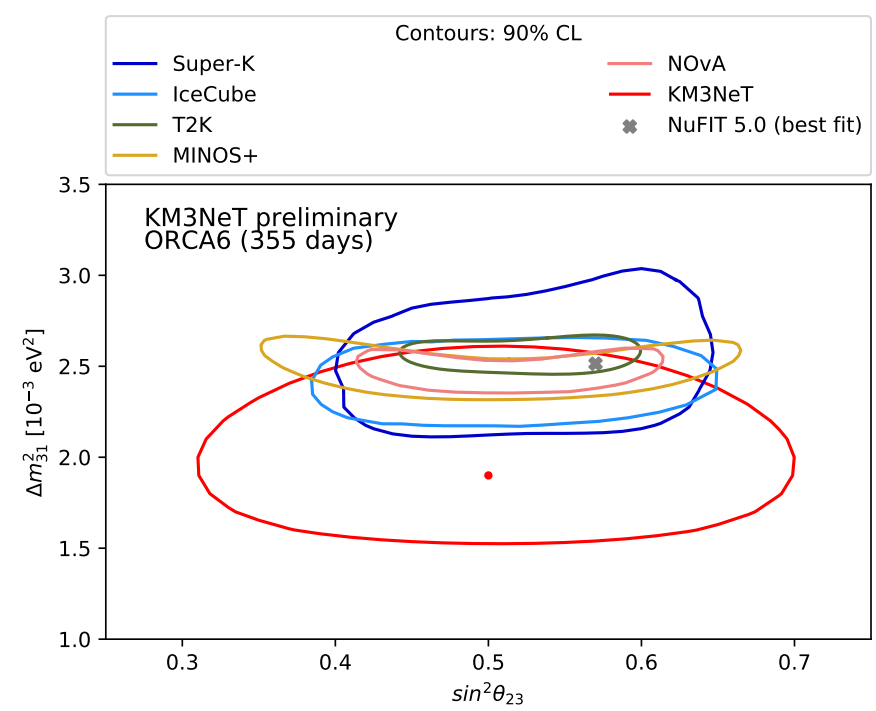

Figure 5: Contour at $90 \% \mathrm{CL}$ of ORCA6 towards the oscillation parameters $\Delta m_{31}^{2}$ and $\theta_{23}$, with the latter shown in the $\sin ^{2}$ basis. Contours of other experiments have been added for comparison purposes as well as the $\mathrm{Nu}$-Fit best fit value [1].

\section{Summary and discussion}

The latest results of ORCA for neutrino oscillation studies has been shown. Using a fit in $\left(E_{\text {reco, }}\right.$, $\left.\cos \theta_{\text {reco }}\right)$ a $\Delta \chi^{2}$ value can be determined that prefers oscillations with $5.9 \sigma$. This is illustrated in terms of L/E. Similarly from profiled likelihood scans in the oscillation parameters $\sin ^{2} \theta_{23}$ and $\Delta m_{31}^{2}$ show clear rejection of a "no oscillations" hypothesis. Finally, the sensitivity of the ORCA6 data set to determine the oscillation parameters is presented with full systematic treatment. From 
this result it is clear that ORCA6 is becoming competitive after almost one year of data taking.

The current analysis does not incorporate the shower channel yet, and improvements to the used energy estimator have been identified that still need to be implemented. As the detector size will increase in the future, the amount of light captured will increase, resulting in a better determination of the energy measurement. Both of these changes will allow for better energy estimation and thus better estimation of $\Delta m_{31}^{2}$. This means there is ample room for improvement. All the while, data taking continues and the detector increases in size.

\section{References}

[1] I. Esteban et al., DOI: 10.1007/JHEP09(2020)178 (2020)

[2] S. Aiello et al., arXiv: 2103.09885 (2021)

[3] S. Adrián-Martínez et al., DOI: 10.1088/0954-3899/43/8/084001 (2016)

[4] S. Aiello et al., DOI: 10.1016/j.cpc.2020.107477 (2020)

[5] G. Carminati et al., DOI: 10.1016/j.cpc.2008.07.014 (2008)

[6] M. Honda et al., DOI: 10.1103/PhysRevD.92.023004 (2015)

[7] G.D. Barr et al., DOI: 10.1103/PhysRevD.74.094009 (2006)

[8] S. Bourret, Neutrino oscillations and earth tomography with KM3NeT-ORCA. Université Sorbonne Paris Cité (PhD Thesis), (2018) 


\section{Full Author List: KM3NeT Collaboration}

M. Ageron ${ }^{1}$, S. Aiello ${ }^{2}$, A. Albert ${ }^{3,55}$, M. Alshamsi ${ }^{4}$, S. Alves Garre ${ }^{5}$, Z. Aly ${ }^{1}$, A. Ambrosone ${ }^{6,7}$, F. Ameli ${ }^{8}$, M. Andre ${ }^{9}$, G. Androulakis ${ }^{10}$, M. Anghinolfi ${ }^{11}$, M. Anguita ${ }^{12}$, G. Anton ${ }^{13}$, M. Ardid ${ }^{14}$, S. Ardid $^{14}$, W. Assal ${ }^{1}$, J. Aublin ${ }^{4}$, C. Bagatelas ${ }^{10}$, B. Baret ${ }^{4}$, S. Basegmez du Pree ${ }^{15}$, M. Bendahman ${ }^{4,16}$, F. Benfenati ${ }^{17,18}$, E. Berbee ${ }^{15}$, A. M. van den Berg ${ }^{19}$, V. Bertin ${ }^{1}$, S. Beurthey ${ }^{1}$, V. van Beveren ${ }^{15}$, S. Biagi ${ }^{20}$, M. Billault ${ }^{1}$, M. Bissinger ${ }^{13}$, M. Boettcher ${ }^{21}$, M. Bou Cabo ${ }^{22}$, J. Boumaaza ${ }^{16}$, M. Bouta ${ }^{23}$, C. Boutonnet ${ }^{4}$, G. Bouvet ${ }^{24}$, M. Bouwhuis ${ }^{15}$, C. Bozza ${ }^{25}$, H.Brânzaş ${ }^{26}$, R. Bruijn ${ }^{15,27}$, J. Brunner ${ }^{1}$, R. Bruno ${ }^{2}$, E. Buis ${ }^{28}$, R. Buompane ${ }^{6,29}$, J. Busto ${ }^{1}$, B. Caiffi ${ }^{11}$, L. Caillat ${ }^{1}$, D. Calvo ${ }^{5}$, S. Campion ${ }^{30,8}$, A. Capone ${ }^{30,8}$, H. Carduner ${ }^{24}$, V. Carretero ${ }^{5}$, P. Castaldi ${ }^{17,31}{ }^{\text {, S. Celli }}{ }^{30,8}$, R. Cereseto ${ }^{11}$, M. Chabab $^{32}$, C. Champion ${ }^{4}$, N. Chau $^{4}$, A. Chen ${ }^{33}$, S. Cherubini ${ }^{20,34}$, V. Chiarella ${ }^{35}$, T. Chiarusi ${ }^{17}$, M. Circella ${ }^{36}$, R. Cocimano $^{20}$, J.A. B. Coelho ${ }^{4}$, A. Coleiro ${ }^{4}$, M. Colomer Molla ${ }^{4,5}$, S. Colonges ${ }^{4}$, R. Coniglione ${ }^{20}$, A. Cosquer ${ }^{1}$, P. Coyle $^{1}$, M. Cresta ${ }^{11}$, A. Creusot ${ }^{4}$, A. Cruz ${ }^{37}$, G. Cuttone ${ }^{20}$, A. D'Amico ${ }^{15}$, R. Dallier $^{24}$, B. De Martino ${ }^{1}$, M. De Palma ${ }^{36,38}$, I. Di Palma ${ }^{30,8}$, A. F. Díaz ${ }^{12}$, D. Diego-Tortosa ${ }^{14}$, C. Distefano ${ }^{20}$, A. Domi ${ }^{15,27}$, C. Donzaud ${ }^{4}$, D. Dornic ${ }^{1}$, M. Dörr ${ }^{39}$, D. Drouhin ${ }^{3,55}$, T. Eberl ${ }^{13}$, A. Eddyamoui ${ }^{16}$, T. van Eeden ${ }^{15}$, D. van Eijk ${ }^{15}$, I. El Bojaddaini ${ }^{23}$, H. Eljarrari ${ }^{16}$, D. Elsaesser ${ }^{39}$, A. Enzenhöfer ${ }^{1}$, V. Espinosa $^{14}$, P. Fermani ${ }^{30,8}$, G. Ferrara ${ }^{20,34}$, M. D. Filipović ${ }^{40}$, F. Filippini ${ }^{17,18}$, J. Fransen ${ }^{15}$, L. A. Fusco ${ }^{1}$, D. Gajanana ${ }^{15}$, T. Gal ${ }^{13}$, J. García Méndez ${ }^{14}$, A. Garcia Soto ${ }^{5}$, E. Garçon ${ }^{1}$, F. Garufi ${ }^{6,7}$, C. Gatius ${ }^{15}$, N. Geißelbrecht ${ }^{13}$, L. Gialanella ${ }^{6,29}$, E. Giorgio ${ }^{20}$, S. R. Gozzini ${ }^{5}$, R. Gracia ${ }^{15}$, K. Graf ${ }^{13}$, G. Grella ${ }^{41}$, D. Guderian ${ }^{56}$, C. Guidi ${ }^{11,42}$, B. Guillon ${ }^{43}$, M. Gutiérrez ${ }^{44}$, J. Haefner ${ }^{13}$, S. Hallmann ${ }^{13}$, H. Hamdaoui ${ }^{16}$, H. van Haren ${ }^{45}$, A. Heijboer ${ }^{15}$, A. Hekalo ${ }^{39}$, L. Hennig ${ }^{13}$, S. Henry ${ }^{1}$, J. J. Hernández-Rey ${ }^{5}$, J. Hofestädt ${ }^{13}$, F. Huang ${ }^{1}$, W. Idrissi Ibnsalih ${ }^{6,29}$, A. Ilioni ${ }^{4}$, G. Illuminati ${ }^{17,18,4}$, C. W. James ${ }^{37}$, D. Janezashvili ${ }^{46}$, P. Jansweijer ${ }^{15}$, M. de Jong ${ }^{15,47}$, P. de Jong ${ }^{15,27}$, B. J. Jung ${ }^{15}$, M. Kadler ${ }^{39}$, P. Kalaczyński ${ }^{48}$, O. Kalekin ${ }^{13}$, U.F. Katz ${ }^{13}$, F. Kayzel ${ }^{15}$, P. Keller ${ }^{1}$, N.R. Khan Chowdhury ${ }^{5}$, G. Kistauri ${ }^{46}$, F. van der Knaap ${ }^{28}$, P. Kooijman ${ }^{27,57}$, A. Kouchner ${ }^{4,49}$, M. Kreter ${ }^{21}$, V. Kulikovskiy ${ }^{11}$, M. Labalme ${ }^{43}$, P. Lagier ${ }^{1}$, R. Lahmann ${ }^{13}$, P. Lamare ${ }^{1}$, M. Lamoureux ${ }^{14}$, G. Larosa ${ }^{20}$, C. Lastoria ${ }^{1}$, J. Laurence ${ }^{1}$, A. Lazo ${ }^{5}$, R. Le Breton ${ }^{4}$, E. Le Guirriec ${ }^{1}$, S. Le Stum ${ }^{1}$, G. Lehaut ${ }^{43}$, O. Leonardi ${ }^{20}$, F. Leone ${ }^{20,34}$, E. Leonora ${ }^{2}$, C. Lerouvillois ${ }^{1}$, J. Lesrel $^{4}$, N. Lessing ${ }^{13}$, G. Levi ${ }^{17,18}$, M. Lincetto ${ }^{1}$, M. Lindsey Clark ${ }^{4}$, T. Lipreau ${ }^{24}$, C. LLorens Alvarez $^{14}$, A. Lonardo $^{8}$, F. Longhitano ${ }^{2}$, D. Lopez-Coto ${ }^{4}$, N. Lumb ${ }^{1}$, L. Maderer ${ }^{4}$, J. Majumdar ${ }^{15}$, J. Mańczak ${ }^{5}$, A. Margiotta ${ }^{17,18}$, A. Marinelli ${ }^{6}$, A. Marini ${ }^{1}$, C. Markou ${ }^{10}$, L. Martin ${ }^{24}$, J. A. Martínez-Mora ${ }^{14}$, A. Martini $^{35}$, F. Marzaioli ${ }^{6,29}$, S. Mastroianni ${ }^{6}$, K. W. Melis ${ }^{15}$, G. Miele ${ }^{6,7}$, P. Migliozzi $^{6}$, E. Migneco ${ }^{20}$, P. Mijakowski ${ }^{48}$, L.S. Miranda ${ }^{50}$, C. M. Mollo ${ }^{6}$, M. Mongelli ${ }^{36}$, A. Moussa ${ }^{23}$, R. Muller ${ }^{15}$, P. Musico ${ }^{11}$, M. Musumeci ${ }^{20}$, L. Nauta ${ }^{15}$, S. $\mathrm{Navas}^{44}$, C. A. Nicolau ${ }^{8}$, B. Nkosi ${ }^{33}$, B. Ó Fearraigh ${ }^{15,27}$, M. O'Sullivan ${ }^{37}$, A. Orlando $^{20}$, G. Ottonello ${ }^{11}$, S. Ottonello ${ }^{11}$, J. Palacios González ${ }^{5}$, G. Papalashvili ${ }^{46}$, R. Papaleo ${ }^{20}$, C. Pastore $^{36}$, A. M. Păun ${ }^{26}$, G. E. Păvălaş ${ }^{26}$, G. Pellegrini ${ }^{17}$, C. Pellegrino ${ }^{18,58}$, M. Perrin-Terrin $^{1}$, V. Pestel $^{15}$, P. Piattelli $^{20}$, C. Pieterse ${ }^{5}$, O. Pisanti ${ }^{6,7}$, C. Poirè ${ }^{14}$, V. Popa ${ }^{26}$, T. Pradier ${ }^{3}$, F. Pratolongo ${ }^{11}$, I. Probst ${ }^{13}$, G. Pühlhofer $^{51}$, S. Pulvirenti ${ }^{20}$, G. Quéméner ${ }^{43}$, N. Randazzo ${ }^{2}$, A. Rapicavoli ${ }^{34}$, S. Razzaque ${ }^{50}$, D. Real ${ }^{5}$, S. Reck ${ }^{13}$,

G. Riccobene ${ }^{20}$, L. Rigalleau ${ }^{24}$, A. Romanov ${ }^{11,42}$, A. Rovelli ${ }^{20}$, J. Royon ${ }^{1}$, F. Salesa Greus ${ }^{5}$, D. F. E. Samtleben ${ }^{15,47}$, A. Sánchez Losa ${ }^{36,5}$, M. Sanguineti ${ }^{11,42}$, A. Santangelo ${ }^{51}$, D. Santonocito ${ }^{20}$, P. Sapienza ${ }^{20}$, J. Schmelling ${ }^{15}$, J. Schnabel ${ }^{13}$, M. F. Schneider ${ }^{13}$, J. Schumann ${ }^{13}$, H. M. Schutte ${ }^{21}$, J. Seneca ${ }^{15}$, I. Sgura ${ }^{36}$, R. Shanidze $^{46}$, A. Sharma ${ }^{52}$, A. Sinopoulou ${ }^{10}$, B. Spisso ${ }^{41,6}$, M. Spurio ${ }^{17,18}$, D. Stavropoulos ${ }^{10}$, J. Steijger $^{15}$, S. M. Stellacci ${ }^{41,6}$, M. Taiuti ${ }^{11,42}$, F. Tatone ${ }^{36}$, Y. Tayalati ${ }^{16}$, E. Tenllado ${ }^{44}$, D. Tézier ${ }^{1}$, T. Thakore ${ }^{5}$, S. Theraube ${ }^{1}$, H. Thiersen ${ }^{21}$, P. Timmer ${ }^{15}$, S. Tingay ${ }^{37}$, S. Tsagkli ${ }^{10}$, V. Tsourapis ${ }^{10}$, E. Tzamariudaki ${ }^{10}$, D. Tzanetatos $^{10}$ C. Valieri ${ }^{17}$, V. Van Elewyck ${ }^{4,49}$, G. Vasileiadis ${ }^{53}$, F. Versari ${ }^{17,18}$, S. Viola ${ }^{20}$, D. Vivolo ${ }^{6,29}$, G. de Wasseige ${ }^{4}$, J. Wilms ${ }^{54}$, R. Wojaczyński ${ }^{48}$, E. de Wolf ${ }^{15,27}$, T. Yousfi ${ }^{23}$, S. Zavatarelli ${ }^{11}$, A. Zegarelli ${ }^{30,8}$, D. Zito $^{20}$, J. D. Zornoza ${ }^{5}$, J. Zúñiga ${ }^{5}$, N. Zywucka ${ }^{21}$.

${ }^{1}$ Aix Marseille Univ, CNRS/IN2P3, CPPM, Marseille, France.

${ }^{2}$ INFN, Sezione di Catania, Via Santa Sofia 64, Catania, 95123 Italy.

${ }^{3}$ Université de Strasbourg, CNRS, IPHC UMR 7178, F-67000 Strasbourg, France.

${ }^{4}$ Université de Paris, CNRS, Astroparticule et Cosmologie, F-75013 Paris, France.

${ }^{5}$ IFIC - Instituto de Física Corpuscular (CSIC - Universitat de València), c/Catedrático José Beltrán, 2 , 46980 Paterna, Valencia, Spain.

\footnotetext{
${ }^{1}$ also at Dipartimento di Fisica, INFN Sezione di Padova and Università di Padova, I-35131, Padova, Italy
} 
${ }^{6}$ INFN, Sezione di Napoli, Complesso Universitario di Monte S. Angelo, Via Cintia ed. G, Napoli, 80126 Italy.

${ }^{7}$ Università di Napoli "Federico II", Dip. Scienze Fisiche "E. Pancini", Complesso Universitario di Monte S. Angelo, Via Cintia ed. G, Napoli, 80126 Italy.

${ }^{8}$ INFN, Sezione di Roma, Piazzale Aldo Moro 2, Roma, 00185 Italy.

${ }^{9}$ Universitat Politècnica de Catalunya, Laboratori d'Aplicacions Bioacústiques, Centre Tecnològic de Vilanova i la Geltrú, Avda. Rambla Exposició, s/n, Vilanova i la Geltrú, 08800 Spain.

${ }^{10}$ NCSR Demokritos, Institute of Nuclear and Particle Physics, Ag. Paraskevi Attikis, Athens, 15310 Greece.

${ }^{11}$ INFN, Sezione di Genova, Via Dodecaneso 33, Genova, 16146 Italy.

${ }^{12}$ University of Granada, Dept. of Computer Architecture and Technology/CITIC, 18071 Granada, Spain. ${ }^{13}$ Friedrich-Alexander-Universität Erlangen-Nürnberg, Erlangen Centre for Astroparticle Physics, ErwinRommel-Straße 1, 91058 Erlangen, Germany.

${ }^{14}$ Universitat Politècnica de València, Instituto de Investigación para la Gestión Integrada de las Zonas Costeras, C/ Paranimf, 1, Gandia, 46730 Spain.

${ }^{15}$ Nikhef, National Institute for Subatomic Physics, PO Box 41882, Amsterdam, 1009 DB Netherlands.

${ }^{16}$ University Mohammed V in Rabat, Faculty of Sciences, 4 av. Ibn Battouta, B.P. 1014, R.P. 10000 Rabat, Morocco.

${ }^{17}$ INFN, Sezione di Bologna, v.le C. Berti-Pichat, 6/2, Bologna, 40127 Italy.

${ }^{18}$ Università di Bologna, Dipartimento di Fisica e Astronomia, v.le C. Berti-Pichat, 6/2, Bologna, 40127 Italy.

${ }^{19}$ KVI-CART University of Groningen, Groningen, the Netherlands.

${ }^{20}$ INFN, Laboratori Nazionali del Sud, Via S. Sofia 62, Catania, 95123 Italy.

${ }^{21}$ North-West University, Centre for Space Research, Private Bag X6001, Potchefstroom, 2520 South Africa.

${ }^{22}$ Instituto Español de Oceanografía, Unidad Mixta IEO-UPV, C/ Paranimf, 1, Gandia, 46730 Spain.

${ }^{23}$ University Mohammed I, Faculty of Sciences, BV Mohammed VI, B.P. 717, R.P. 60000 Oujda, Morocco.

${ }^{24}$ Subatech, IMT Atlantique, IN2P3-CNRS, Université de Nantes, 4 rue Alfred Kastler - La Chantrerie, Nantes, BP 2072244307 France.

${ }^{25}$ Università di Salerno e INFN Gruppo Collegato di Salerno, Dipartimento di Matematica, Via Giovanni Paolo II 132, Fisciano, 84084 Italy.

${ }^{26}$ ISS, Atomistilor 409, Măgurele, RO-077125 Romania.

${ }^{27}$ University of Amsterdam, Institute of Physics/IHEF, PO Box 94216, Amsterdam, 1090 GE Netherlands.

${ }^{28}$ TNO, Technical Sciences, PO Box 155, Delft, 2600 AD Netherlands.

${ }^{29}$ Università degli Studi della Campania "Luigi Vanvitelli", Dipartimento di Matematica e Fisica, viale Lincoln 5, Caserta, 81100 Italy.

${ }^{30}$ Università La Sapienza, Dipartimento di Fisica, Piazzale Aldo Moro 2, Roma, 00185 Italy.

${ }^{31}$ Università di Bologna, Dipartimento di Ingegneria dell'Energia Elettrica e dell'Informazione "Guglielmo Marconi", Via dell'Università 50, Cesena, 47521 Italia.

${ }^{32}$ Cadi Ayyad University, Physics Department, Faculty of Science Semlalia, Av. My Abdellah, P.O.B. 2390, Marrakech, 40000 Morocco.

${ }^{33}$ University of the Witwatersrand, School of Physics, Private Bag 3, Johannesburg, Wits 2050 South Africa.

${ }^{34}$ Università di Catania, Dipartimento di Fisica e Astronomia "Ettore Majorana", Via Santa Sofia 64, Catania, 95123 Italy.

${ }^{35}$ INFN, LNF, Via Enrico Fermi, 40, Frascati, 00044 Italy.

${ }^{36}$ INFN, Sezione di Bari, via Orabona, 4, Bari, 70125 Italy.

${ }^{37}$ International Centre for Radio Astronomy Research, Curtin University, Bentley, WA 6102, Australia.

${ }^{38}$ University of Bari, Via Amendola 173, Bari, 70126 Italy.

${ }^{39}$ University Würzburg, Emil-Fischer-Straße 31, Würzburg, 97074 Germany.

${ }^{40}$ Western Sydney University, School of Computing, Engineering and Mathematics, Locked Bag 1797, Penrith, NSW 2751 Australia.

${ }^{41}$ Università di Salerno e INFN Gruppo Collegato di Salerno, Dipartimento di Fisica, Via Giovanni Paolo II 132, Fisciano, 84084 Italy.

${ }^{42}$ Università di Genova, Via Dodecaneso 33, Genova, 16146 Italy.

${ }^{43}$ Normandie Univ, ENSICAEN, UNICAEN, CNRS/IN2P3, LPC Caen, LPCCAEN, 6 boulevard Maréchal 
Juin, Caen, 14050 France.

${ }^{44}$ University of Granada, Dpto. de Física Teórica y del Cosmos \& C.A.F.P.E., 18071 Granada, Spain.

${ }^{45}$ NIOZ (Royal Netherlands Institute for Sea Research), PO Box 59, Den Burg, Texel, 1790 AB, the Netherlands.

${ }^{46}$ Tbilisi State University, Department of Physics, 3, Chavchavadze Ave., Tbilisi, 0179 Georgia.

${ }^{47}$ Leiden University, Leiden Institute of Physics, PO Box 9504, Leiden, 2300 RA Netherlands.

${ }^{48}$ National Centre for Nuclear Research, 02-093 Warsaw, Poland.

${ }^{49}$ Institut Universitaire de France, 1 rue Descartes, Paris, 75005 France.

${ }^{50}$ University of Johannesburg, Department Physics, PO Box 524, Auckland Park, 2006 South Africa.

${ }^{51}$ Eberhard Karls Universität Tübingen, Institut für Astronomie und Astrophysik, Sand 1, Tübingen, 72076 Germany.

52 Università di Pisa, Dipartimento di Fisica, Largo Bruno Pontecorvo 3, Pisa, 56127 Italy.

${ }^{53}$ Laboratoire Univers et Particules de Montpellier, Place Eugène Bataillon - CC 72, Montpellier Cédex 05, 34095 France.

${ }^{54}$ Friedrich-Alexander-Universität Erlangen-Nürnberg, Remeis Sternwarte, Sternwartstraße 7, 96049 Bamberg, Germany.

${ }^{55}$ Université de Haute Alsace, 68100 Mulhouse Cedex, France.

${ }^{56}$ University of Münster, Institut für Kernphysik, Wilhelm-Klemm-Str. 9, Münster, 48149 Germany.

${ }^{57}$ Utrecht University, Department of Physics and Astronomy, PO Box 80000, Utrecht, 3508 TA Netherlands.

${ }^{58}$ INFN, CNAF, v.le C. Berti-Pichat, 6/2, Bologna, 40127 Italy. 